\title{
Cartilage and bone damage in rheumatoid arthritis
}

\author{
Monika Ostrowska ${ }^{1}$, Włodzimierz Maśliński ${ }^{2}$, Monika Prochorec-Sobieszek ${ }^{3,4}$, Michał Nieciecki ${ }^{5,6}$, \\ Iwona Sudoł-Szopińska ${ }^{1,5}$ \\ ${ }^{1}$ Department of Radiology, National Institute of Geriatrics, Rheumatology and Rehabilitation, Warsaw, Poland \\ 2Department of Pathophysiology and Immunology, National Institute of Geriatrics, Rheumatology and Rehabilitation, Warsaw, Poland \\ ${ }^{3}$ Diagnostic Haematology Department, Institute of Haematology and Transfusion Medicine, Warsaw, Poland \\ ${ }^{4}$ Department of Pathomorphology, National Institute of Geriatrics, Rheumatology and Rehabilitation, Warsaw, Poland \\ ${ }^{5}$ Department of Diagnostic Imaging, Medical University of Warsaw, Poland \\ ${ }^{6}$ Department of Nuclear Medicine, Medical University of Warsaw, Poland
}

\begin{abstract}
Rheumatoid arthritis (RA), which is a chronic inflammatory disease with a multifactorial aetiology, leads to partial or permanent disability in the majority of patients. It is characterised by persistent synovitis and formation of pannus, i.e. invasive synovial tissue, which ultimately leads to destruction of the cartilage, subchondral bone, and soft tissues of the affected joint. Moreover, inflammatory infiltrates in the subchondral bone, which can lead to inflammatory cysts and later erosions, play an important role in the pathogenesis of RA. These inflammatory infiltrates can be seen in magnetic resonance imaging (MRI) as bone marrow oedema (BME). BME is observed in $68-75 \%$ of patients in early stages of RA and is considered a precursor of rapid disease progression. The clinical significance of synovitis and bone marrow oedema as precursors of erosions is well established in daily practice, and synovitis, BME, cysts, hyaline cartilage defects and bone erosions can be detected by ultrasonography (US) and MRI. A less explored subject is the inflammatory and destructive potential of intra- and extra-articular fat tissue, which can also be evaluated in US and MRI. Finally, according to certain hypotheses, hyaline cartilage damage may trigger synovitis and lead to irreversible joint damage, and MRI may be used for preclinical detection of cartilage biochemical abnormalities.

This review discusses the pathomechanisms that lead to articular cartilage and bone damage in RA, including erosion precursors such as synovitis and osteitis and panniculitis, as well as the role of imaging techniques employed to detect early cartilage damage and bone erosions.
\end{abstract}

Key words: rheumatoid arthritis, synovitis, erosions, cartilage, magnetic resonance imaging, ultrasound.

\section{Introduction}

Rheumatoid arthritis (RA), the most common inflammatory rheumatic disease, may lead to severe joint destruction and disability. While the involvement of cartilage and bone in RA is undisputed, the corresponding pathogenesis is subject to ongoing discussion. The mechanism, where the fibroblast-like synoviocytes initiate cartilage and bone damage through direct invasion and trigger catabolic cascades, has been termed the outside-in hypo- thesis [1]. There is also evidence supporting an osteitiscentred approach, referred to as the inside-out hypothesis, in which inflammation and joint damage originate from the bone tissue [1].

Another tissue involved in cartilage and bone damage in RA is extra- or intra-articular fat tissue [2]. It has been confirmed that adipose tissue is infiltrated by inflammatory cells and, moreover, it itself produces ca. 50 biologically active factors called adipo(cyto)kines, and therefore it may be involved in the degradation of 
all connective tissue components, including cartilage. Abnormal fat tissue echogenicity or signals are well observed in ultrasound (US) and magnetic resonance imaging (MRI). Finally, according to certain hypotheses, hyaline cartilage damage may activate and maintain synovitis [3]. Quantitative MR applications to cartilage are able to show glycosaminoglycan loss, which possibly precedes visible cartilage damage [4] and might identify early cartilage pathology before more severe morphological changes occur.

\section{Pathomechanisms leading to articular cartilage and bone damage in rheumatoid arthritis}

\section{The synovium and the pannus}

Macroscopically, the synovial membrane (the synovium) is a thin layer of connective tissue that lines the internal surface of joint capsules, tendon sheaths and bursae. Its main function is to nourish joint cartilage by producing joint fluid rich in hyaluronic acid $[5,6]$. The synovium is composed of two layers: the more superficial intima layer and the subintima layer below it. The intima contains only 1-3 layer(s) of cells and lacks a basement membrane, which facilitates the flow of joint fluid between the vessels in the subintima and the joint space. It is composed of extracellular matrix proteins and synoviocytes [5]. Mesenchymal fibroblast-like synoviocytes (FLS) prevail, while macrophage-like synoviocytes (MfLS) are less numerous. It is known that both types of synoviocytes are the main sources of factors responsible for inflammatory and destructive processes in joints, such as pro-inflammatory cytokines, chemok-

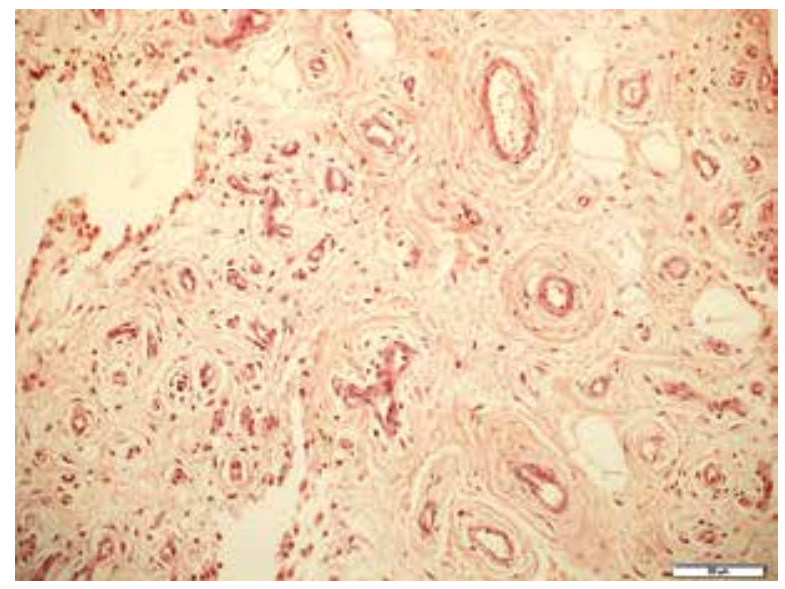

Fig. 1. Angiogenesis in the synovium of an rheumatoid arthritis patient with numerous proliferating vessels seen in hypertrophied villi. H\&E, × 200 . ines, and enzymes degrading all connective tissue components, including cartilage and subchondral bone [6].

The mechanism of joint inflammation and destruction that begins in the synovium is called the outside-in hypothesis [1]. It starts with the transformation of the normal synovium to invasive pannus through the thickening of both the intima and subintima layers of the synovium. This stage of RA is already clearly seen in US and MRI. The intima is thickened through mild hyperplasia (increased number of cells from 1-3 layers up to 8-10 layers) and subintima through the migration and retention of infiltrating cells [6]. Cellular infiltrates in the subintima form ectopic lymphoid tissue, which is a site for a local autoimmune response. An indisputable sign of invasive synovial tissue, called pannus, is increased synovial vascularity, which results from neoangiogenesis [6] (Fig. 1). This stage of the disease is visible in both power Doppler (PD) or colour Doppler (CD) US and in MRI, particularly on contrast-enhanced T1-weighted images, which show an increased signal from the thickened and vascularised synovium [6].

In RA, the synovial fluid is the primary "compartment" that degrades cartilage and bone by a mechanism involving synergistic interaction between cytokines and some other components of synovial fluid. The main stimuli of cartilage degradation are thought to be two cytokines detected in RA synovial fluid: interleukin 1 (IL-1) and tumour necrosis factor (TNF) [7]. In vitro studies confirmed that $\mathrm{IL}-1$ and TNF can degrade cartilage by stimulating chondrocytes to secrete cartilage-degrading metalloproteases which are then activated by other synovial fluid enzymes, leading to glycosaminoglycan loss. Another hypothesis states that synovial fluid sensitises chondrocytes to IL-1 and TNF [7]. The second mechanism (tissue) destroying cartilage is the above-described pannus, which directly infiltrates cartilage and soft tissues of the joint, according to the so-called outside-in hypothesis [1].

\section{Bone marrow inflammation}

Clumps of ectopic lymphatic tissue that lead to cartilage and bone destruction do not form only in the synovium, but also in the subchondral bone marrow [8-10]. This is seen in MRI as BME, which is detected in as many as $68-75 \%$ of patients in early phases of RA [11] and predicts rapid development of RA with 100\% accuracy [12]. BME has a number of other important diagnostic and prognostic characteristics [13-16]:

- it appears several weeks after the first symptoms, thus being considered a very early marker of inflammation,

- it is the most significant and sensitive MRI finding in RA patients, particularly in the early phase of RA (lasting less than 1 year), 
- it may develop independently of synovitis,

- it is considered a forerunner of erosions; the risk of erosion formation is 6-fold higher in areas where BME has been noted,

- it is twice as common in early RA patients with anticitrullinated antibodies (ACPA), a finding supporting its association with a rapid and aggressive course of the disease,

- it is reversible and can be reduced with biological treatment, for example anti-TNF $\alpha$ drugs; erosions do not form in $50 \%$ of patients with BME thanks to early and appropriate therapy.

Histopathology has shown that all areas of BME result from the substitution of the bone marrow for inflammatory infiltrates containing macrophages, memory $T$ cells, B cells, plasma cells and osteoclasts - all in all, a picture consistent with bone inflammation (osteitis or osteomyelitis) [13]. Jimenez-Boy et al. [10] provided histopathological evidence for direct penetration of inflammatory synovial tissue through the cortical bone barrier and induction of inflammatory changes in the adjacent bone marrow. There is also evidence that proinflammatory cytokines, such as TNF, IL-1 and IL-6, strongly inhibit the differentiation of multipotent mesenchymal stem cells to adipocytes, which are abundant in the bone marrow. Therefore, in addition to inflammatory cells, the increased number of undifferentiated mesenchymal cells is behind the MRI detectable bone marrow edema.

Bone erosions may develop as a result of cytokines released by inflammatory cells in the marrow, including RANKL, a cytokine produced by osteocytes, T and B lymphocytes and FLS, which stimulate osteoclasts for bone resorption, thus leading first to the formation of an inflammatory cyst and afterwards to perforation of the cortical layer and cartilage with the subsequent development of erosions and accumulation of inflammatory cells within the synovium.

These inflammatory cells from the bone marrow may also reach the synovium directly, even before cortical layer disruption, through bone canaliculi, or indirectly, through the peripheral blood after passing between the walls of the synovial blood vessels [13]. They mediate erosions from the synovial membrane inwards as the main sources of potentially pathological cells which carry out their effector functions from the side of the subchondrium in the above-mentioned mechanism, known as the inside-out or bone-marrow-centred disease model for RA development $[13,17]$. The bone-marrow-model could explain the success of drugs such as rituximab, aimed at B cells, which may reside in the synovium but originate from the bone marrow [17].
The above-presented mechanisms of bone and cartilage damage in RA could be summarised as follows $[17,18]$ :

1. The pannus directly leads to the development of erosions (outside-in model).

2. Inflammation of the bone marrow may: a) on the one hand, cause subcortical bone destruction, initially presenting as an inflammatory cyst and later as an erosion on radiographs, in US and MRI; and b) on the other hand, result in the migration of inflammatory mediators and cells into the joint cavity through enlarged channels within bone tissue, or with circulation, leading to synovitis, and in an indirect manner, to erosions (inside-out model).

And finally, another factor originating from bone tissue and impairing cartilage quality is angiogenesis, which adversely affects cartilage biomechanical properties. Healthy cartilage does not possess vessels and even releases an array of anti-angiogenic factors [19]. In RA (as in osteoarthritis, OA), the developing pathologic vessels infiltrate the cartilage from the side of the bone, probably due to the imbalance between pro- and anti-angiogenic factors [19].

\section{The rheumatoid adipose tissue}

In RA and OA, fat tissue has been found to be infiltrated by inflammatory cells, mainly leukocytes (i.e. monocytes/macrophages), neutrophils, basophils, eosinophils and lymphocytes (Fig. 2). In pathological conditions, $\mathrm{T}$ and $\mathrm{B}$ cells, natural killers and mastocytes are also found [2]. In addition to being infiltrated by a number of inflammatory cells, adipose tissue produces ca. 50 biologically active factors called adipocytokines (or adipokines) which include, among others, proinflammatory cytokines

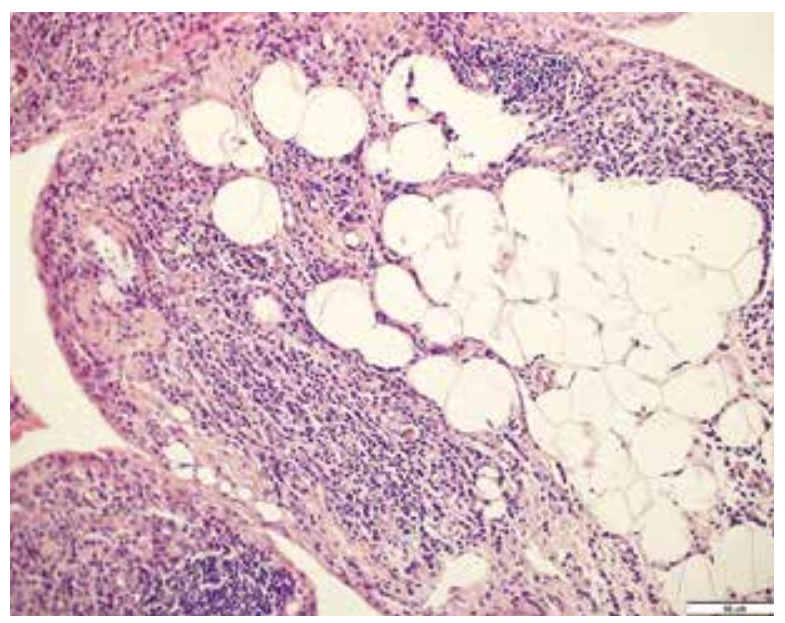

Fig. 2. Inflammatory infiltrates in the adipose tissue. $H \& E, \times 200$. 
and other inflammatory mediators (such as $C$ reactive protein - CRP, serum amyloid - SAA, prostaglandin E2 and nitric oxide). In addition, fat tissue secrets conventional adipokines - leptin, adiponectin and visfatin [20].

By secreting those factors, the fat tissue induces the production of matrix metalloproteinases (MMP), which may degrade the cartilage and all other components of the connective tissue [21].

\section{Cartilage as the primary source of inflammation}

The traditional paradigm presented above, namely that the synovium and subchondral bone marrow inflammation lead to characteristic cartilage destruction in RA, as noted by Smolen et al. [3], is not fully true. Also in other diseases, such as reactive or psoriatic arthritis, synovitis is histologically similar to RA (although without eliciting a similar degree of joint destruction as in RA), with the same cytokines upregulated and the same response to therapies targeting cytokines [3]. Instead, it has long been known that immune complexes are deposited in RA cartilage, and that they may contain rheumatoid factor (RF), ACPA, collagen and anti-RA33 antibodies, which are also typically associated with RA and which may also be present in the circulation and in joint fluids. Moreover, it has been discovered that collagen and other cartilage constituents induce experimental arthritis and activate the inflammatory response in RA [3]. So, there is probably an additional mechanism in RA where cartilage autoantigens activated by joint/ cartilage damage cause chronic synovitis [3]. To support this hypothesis, experimental models of arthritis would be needed for example to show that microsurgical elimination of a cartilage defect prevents collagen arthritis or other forms of experimental inflammatory joint disease [3]. Such models could engage quantitative MR applications to cartilage, such as dGEMRIC or T2 mapping, which in research studies were proved to detect early arthritic changes in cartilage before the occurrence of more severe morphological changes.

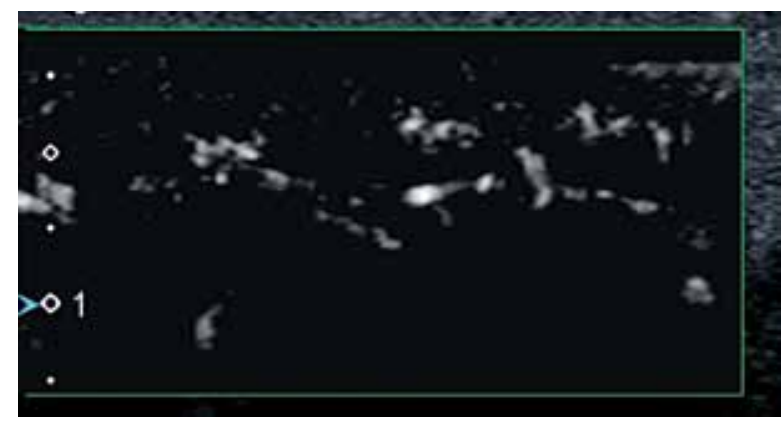

\section{The role of imaging in the detection of early cartilage damage and bone erosions}

In RA, most intensive damage to the joints occurs in the first 2 years, and early progression is associated with poor outcomes. Thus, it is of utmost importance to diagnose the disease early, and implement treatment to prevent destruction [16]. Findings on plain radiography are normal in the majority of patients at the time of diagnosis, and radiographic changes are often delayed by 6-12 months after RA onset [16].

The two most commonly used imaging methods to diagnose early RA are US and MRI. Because of their excellent soft tissue contrast, they can depict RA at a very early stage. Ultrasonography is mainly used to identify and monitor synovitis during treatment. By contrast with ultrasound, MRI is capable of visualising all anatomical components of joints involved in RA down to the molecular level, including bone and cartilage. MRI can therefore depict imaging features of all pathogenetic hypotheses mentioned above [1].

\section{Imaging of synovitis, panniculitis, osteitis, and cartilage defects}

The thickened and vascularised pannus and its destructive effects on joint cartilage and subchondral bone can be seen in US, including PD and CD, and even in more sensitive US techniques of low-velocity microvascular flow detection (Fig. 3). Due to limited access to many surfaces, US can show only some of the inflammatory cysts and erosions, and partially the pannus. Regarding hyaline cartilage, although its evaluation in US is limited to some surfaces, US may visualise increased echogenicity of cartilage and/or its ill-defined outlines, representing an early stage of cartilage degeneration (Fig. 4). And finally, intra- or extra-articular fat tissue abnormalities, which frequently accompany joint diseases, may be easily seen (Fig. 5).

By contrast with US, MRI evaluates all joint parts and may identify synovitis, consecutive stages of cartilage loss, bone erosions, BME and inflammatory cysts [4]

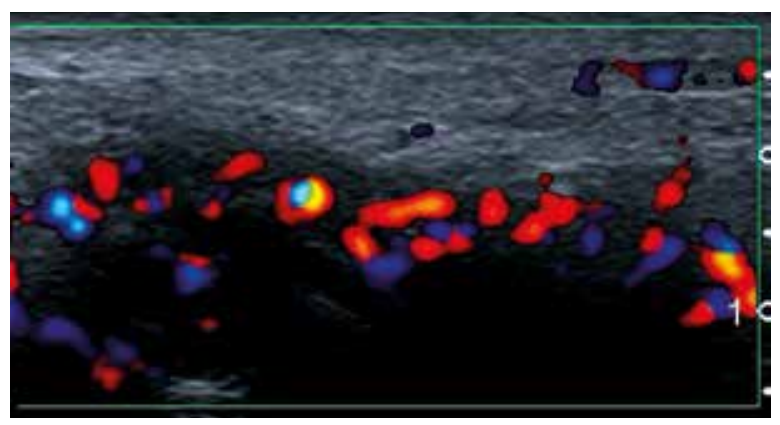

Fig. 3. Ultrasound of the hand: metacarpophalangeal joints 2 joint synovitis, evident in both colour Doppler and low-velocity microvascular flow detection scan (left part of the image). 
(Figs. 6, 7). Abnormal synovium is thickened, has intermediate to low signal intensity on T1-weighted (w) images and a high signal on T2w, PD fat-suppressed (FS), STIR images, and shows quick enhancement after administration of contrast media. BME has a high signal on T2 FS, PD FS and STIR images and presents ill-defined margins. It is hypointense and less evident in T1w images and becomes enhanced (hyperintense) after contrast administration, best seen on T1 FS images [22].

Contrast injection is not obligatory to neither visualise BME nor to increase the specificity of that finding. Regardless of the origin (e.g. inflammation, infection, mechanical load, trauma, tumour), BME shows enhancement on post-contrast images. Subchondral cysts are identical to the image of BME, but they present as more focal lesions located in the vicinity of the bone cortex $[13,22]$. Erosions are sharply marginated defects within the trabecular bone with disrupted cortical bone. They are characterised by low signal intensity on T1w images, high signal intensity on T2, PD FS and STIR images, and enhancement upon contrast administration. Cartilage loss is clearly visible on PD and T2w images, also with fat saturation on PD FS and T2 FS images.

In some centres the modified Outerbridge system (grading scale) introduced in 2006, which originally was based on arthroscopic findings, is used to reflect changes in hyaline cartilage on MRI $[23,24]$. This system is based on grading of the depth, location, and severity of chondral injuries as follows: grade 0 - normal cartilage, grade 1 signal intensity alterations with an intact surface of the articular cartilage compared with the surrounding normal cartilage, grade 2 - partial thickness defect of the cartilage, grade 3 - fissuring of the cartilage to the level of the subchondral bone and grade 4 - exposed subchondral bone.

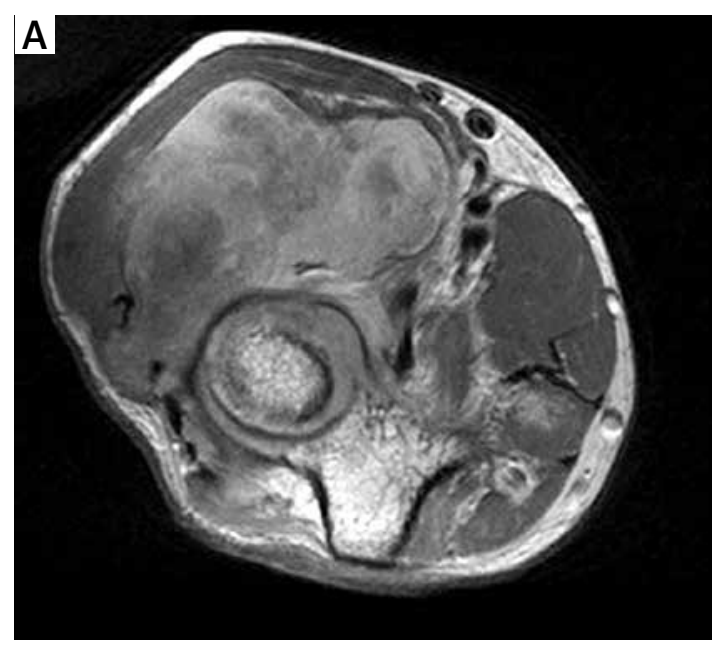

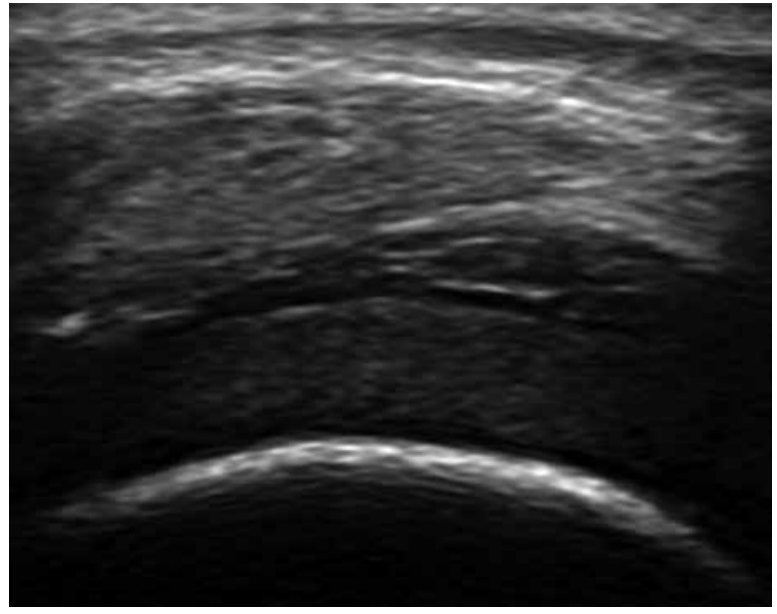

Fig. 4. Knee joint hyaline defect in a 17-year-old adolescent with juvenile idiopathic arthritis presenting with increased echogenicity of cartilage, despite smooth outline.

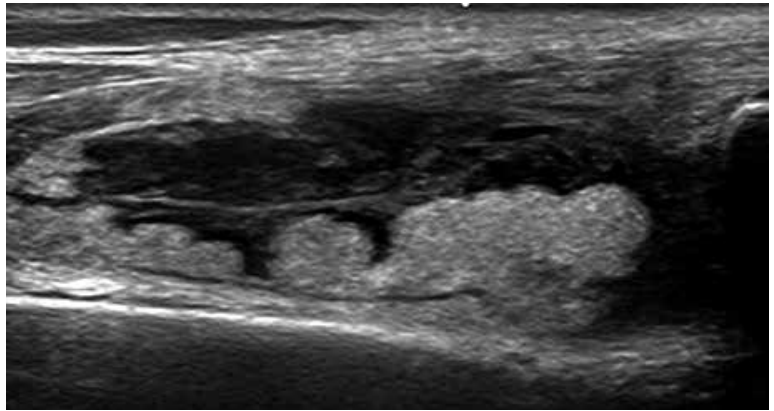

Fig. 5. Oedema of the prefemoral fat pad of the knee joint in a 64-year-old patient with rheumatoid arthritis. Thickened hypoechoic synovium is seen superficial to thickened fat tissue.

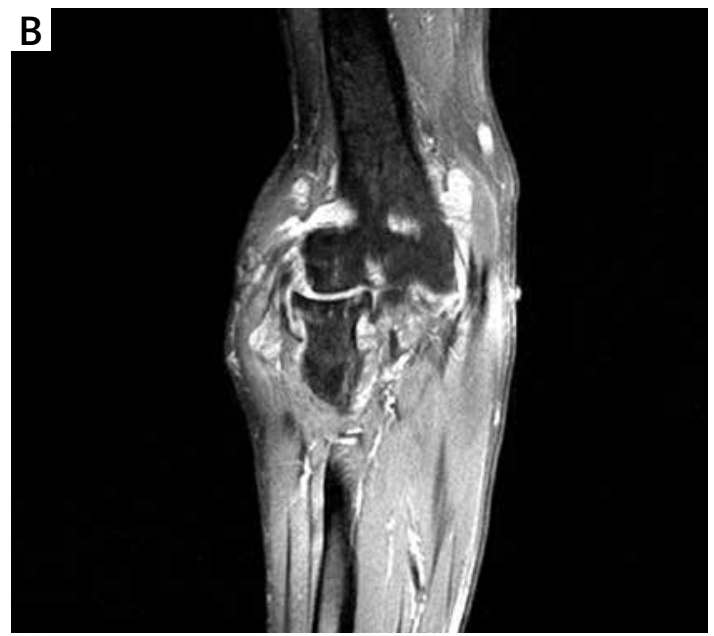

Fig. 6. Magnetic resonance imaging of the right elbow: axial PD (A), coronal T1 FS contrast medium (CM): effusions, significant thickening and post-contrast enhancement of the synovium seen as a lobulated, polycyclic mass, distending the capsule and penetrating between extensor and flexor muscles (B); cysts, hyaline cartilage loss in the lateral part and erosions in the medial part of the elbow joint. 

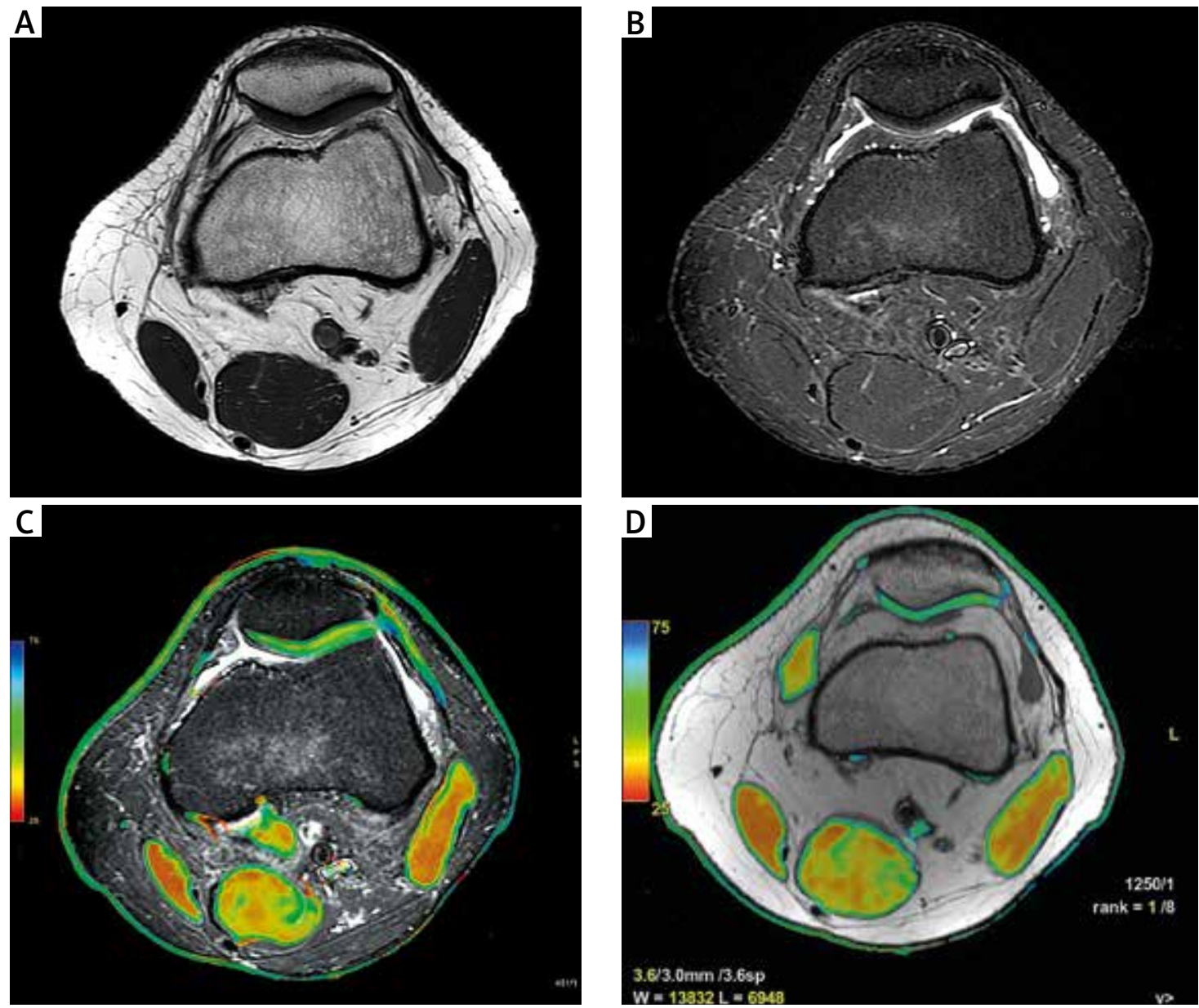

Fig. 7. Magnetic resonance imaging of the knee in a 19-year-old man with RA: axial PD (A), axial T2 TIRM (B), axial T2 TIRM with overlapped T2 mapping (C), axial T2 mapping: focal chondromalacia on the lateral surface of the patella (D); yellow colour on T2 mapping indicates cartilage degeneration.

The most sensitive methods to identify small, shallow erosions/irregularities are arthroCT and arthroMR. However, as these procedures carry the risk of joint inflammation, they are performed only in doubtful cases. Erosions are clearly seen also in the case of joint effusion.

\section{Biochemical and metabolic imaging of cartilage in rheumatoid arthritis}

Morphological imaging of cartilage is possible mainly in PD, PD FS/SPAIR, T1 VISTA (3D), and 3D FSPGR sequences. It has been shown that in the early stage of $\mathrm{OA}$ and other hyaline cartilage diseases, proteoglycans (PGs) and glycosaminoglycans (GAGs) leak from cartilage, and collagen fibres change in size and orientation, allowing more water and less restricted water diffusion into the cartilage [25]. These initial disease processes occur without evident cartilage defect [25]. When a cartilage disease progresses, morphologic changes (thinning and defects) appear.
Magnetic resonance and $\mathrm{CT}$ have recently been introduced as tools enabling detection of cartilage biochemical abnormalities before morphological changes occur and providing quantitative measures that correlate with collagen and $P G$ content $[25,26]$. Some MR and CT techniques correlate with the GAG component of PGs (dGEMRIC, sodium MRI), whereas other techniques correlate with water content (T1rho mapping), or water and collagen content and orientation (T2 mapping and $\mathrm{T}^{\star}$ mapping) (Fig. 7) or analyse cartilage only (ultrashort echo time - UTE, gagCEST) [23]. Although they are still evolving and have disadvantages, including lengthened scan times, required special hardware and low spatial resolution, experimental studies are promising and provide different outcome measures that can be used as imaging biomarkers [25-29].

To date, the most studied and promising in RA is delayed gadolinium-enhanced MRI of cartilage (dGEMRIC), in which the contrast accumulates in cartilage inversely 
to the GAG content $[25,30-32]$. The outcome parameter is $T 1$ relaxation time ( $T 1_{G d}$ in milliseconds). Because gadolinium reduces $T 1$ relaxation time, longer $T 1_{G d}$ values are obtained from healthy cartilage, whereas lower $\mathrm{T} 1_{G d}$ values indicate cartilage degeneration and a GAG decrease early in the process of cartilage degeneration $[30,33]$. In RA, dGEMRIC has been used to visualise early cartilage damage in finger joints and to assess the therapeutic effect of TNF inhibitors [25].

Schleich et al. [30] showed that high MRI inflammatory scores in dGEMRIC were associated with cartilage proteoglycan loss at the patient level. The joint with a higher RAMRIS synovitis subscore demonstrated a significantly lower dGEMRIC value in the intra-individual analysis, representing a higher degree of cartilage destruction. Interestingly, Tiderius et al. demonstrated that cartilage damage in biochemical MRI continued irrespective of the disease activity following therapy escalation with TNF-alpha-blockers [34].

Müller-Lutz et al. [35] investigated the correlation between semiquantitative and quantitative dynamic contrast-enhanced (DCE) parameters with dGEMRIC of the metacarpophalangeal joints (MCP) of patients with early RA using a 3-T MRI scanner. Significant correlations were noted between DCE parameters and the RA MRI score of the second MCP joint. There was a significant negative correlation between DCE parameters and dGEMRIC, and no association between DCE parameters and joint space width. The authors concluded that semiquantitative and quantitative analyses of perfusion are applicable to show that cartilage damage correlates with inflammation activity despite the absence of joint space narrowing [35].

Miese et al. [36] evaluated finger joint cartilage degeneration in RA with dGEMRIC using dedicated surface coils for increased spatial resolution. They wanted to confirm the findings of Smolen, who hypothesised that cartilage damage promotes joint inflammation via liberation of neoepitopes and subsequent perpetuation of the immune response [3]. They did not reveal an association between $\mathrm{dGEMRIC}$ index and serologic markers of RA such as ACPA, which are known to be predictive of joint damage [36]. The dGEMRIC index was independent of systemic inflammation (as measured by CRP level) and disease activity (as measured by the DAS28) [36].

DGEMRIC is regarded as the best available imaging tool for indirect GAG measurement in vivo. However, the main disadvantage is long examination time because of the interval needed between contrast injection and scanning. Finally, not only cartilage amount is reflected by the contrast accumulation, but also other factors may play role, such as collagen content and/or orientation, duration and type of examination [25].
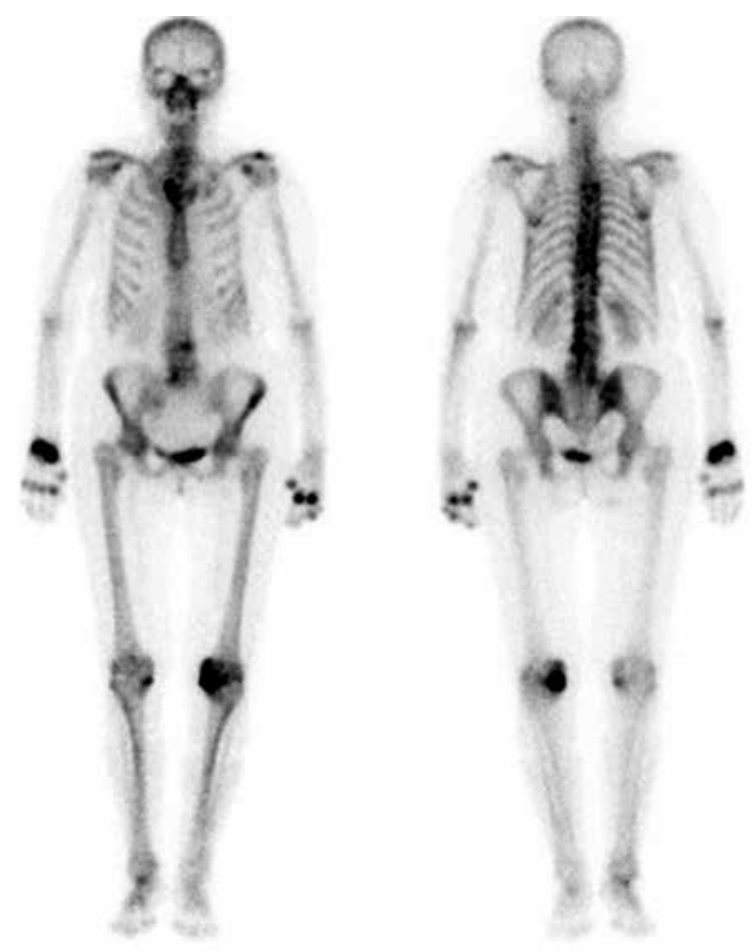

Fig. 8. Planar whole-body delayed-phase ${ }^{99 \mathrm{~m} T c-M D P}$ bone scan in the anterior and posterior projections in a 65-year-old woman with rheumatoid arthritis: increased uptake in the spine and multiple joints: right sternoclavicular, in multiple distal and proximal interphalangeal joints, metacarpophalangeal joints and right wrist, as well as left knee.

With regard to other cartilage-specific MRI techniques, Buchbender et al. [31] compared T2* and native T1 mapping with dGEMRIC. They were both of low diagnostic value compared to dGEMRIC in RA patients. Pre-contrast T1 mapping used for the calculation of $\Delta R 1$ was also time-consuming, often painful in RA, and did not increase the diagnostic value of dGEMRIC.

Additional information may be obtained from functional imaging techniques that collect metabolic information from tissues involved in inflammatory processes in RA [37]. Histopathological evaluation of the tissue obtained from joints of RA patients showed significant accumulation of osteoblasts adjacent to focal bone erosions and sites exposed to inflammatory synovial tissue [1]. Traditionally, increased metabolic activity of bone tissue may be evaluated by planar scintigraphy with technetium methylene diphosphonate ( ${ }^{99 m}$ Tc MDP) (Fig. 8).

Radiotracer accumulation indicates increased osteogenic activity in the areas of bone formation or repair, e.g. due to inflammation. In a three-phase dynamic bone 
scan, ${ }^{99 m}$ Tc-MDP is administered as a bolus injection. In the first phase, a sequence of 2 - to 5 -second images is obtained for 1 minute after radiotracer injection. It is followed by the blood pool or soft tissue phase consisting of static images acquired for several minutes. Skeletal phase (delayed) images are obtained at 2 to 4 hours. Typically, the whole-body scan is performed with additional spot views of suspicious areas.

In order to improve the quality of bone imaging, three-dimensional single photon emission computed tomography (SPECT) has been introduced. The inflammatory response of bone tissue can be precisely visualised, for instance with technetium-99m-labelled disphosphonates (Tc-99m DPD) [37]. Other modifications include multi pinhole collimator views (multi-pinhole SPECT; MPH-SPECT) and fusion of MRI and SPECT, which offers high-contrast images of very initial bone alterations in RA [37]. The utility of bone SPECT and MRI in the assessment of patients with RA has been frequently compared. Several studies indicated that increased uptake of Tc99m-DPD in patients with RA was seen in the MCP joints despite the lack of BME. Furthermore, these joints later showed erosive progression. Thus, hybrid MPH-SPECT and MRI might provide valuable additional information for identification of RA patients with a high risk of erosive progression [37].

The same authors also noted that the MCP joints which showed progression of the size of erosions or new erosion formation had a significantly higher ${ }^{99 \mathrm{~m} T \mathrm{TC}-\mathrm{DPD}}$ uptake in baseline SPECT compared to joints with stable or regressive erosions [37]. These findings suggested that increased ${ }^{99 \mathrm{~m} T c-D P D}$ as a measure of increased bone metabolism might be predictive of joint erosion progression in RA. Yet another nuclear medicine imaging technique, namely positron emission tomography (PET), most commonly used with ${ }^{18} \mathrm{~F}$-fluorodeoxyglucose ( $\left.{ }^{18} \mathrm{~F}-\mathrm{FDG}\right)$, is also considered to be a sensitive method to visualise inflammatory changes in RA patients, even for subclinical arthritis. This method has also been used for therapy monitoring [37].

\section{Discussion and future perspectives}

MRI has the ability to early diagnose RA when it is still masked to conventional radiography, by direct visualisation of the rheumatoid synovium [16] and, which is even more important, by bone marrow oedema. BME is seen by MRI only and has been shown to be a strong predictor of bone and cartilage damage in RA. MRI can be used for early diagnosis and treatment follow-up.

For rheumatologists, synovitis remains the most important indicator of an aggressive course of the disease. However, this element of RA has not been confirmed to be an independent predictor of joint destruction in several studies. They showed that isolated synovitis is not a predisposing factor for the development of erosions; thus the prognosis of these patients could be better [37]. It is possible that such patients may not require as aggressive treatment as do those with BME. In addition, an MRI-, CT- and US-controlled study on a larger RA patient cohort receiving a combined adalimumab and methotrexate treatment reported no progression and even occasional healing of joint erosions despite persisting synovitis [37].

Hyaline cartilage is emerging as another site of early RA location. Biochemical and molecular cartilage MRI allows imaging deep into the cartilage structure. Owing to this, it is also possible to undertake experimental studies, as hypothesised by Smolen et al. [3], aimed at evaluating the relation between cartilage damage and joint disease. Several of these imaging modalities can be used with regular MRI machines, using a contrast agent also employed on a routine basis [25]. The acquisition time is not much longer than in standard morphological sequences. However, image post-processing is still fairly sophisticated and time-consuming, which prevents these new and promising techniques from becoming incorporated into routine clinical practice yet [25].

Currently, apart from single publications, we do not take the advantage of cartilage MRI seriously and concentrate traditionally on BME, pannus and erosion detection. However, the understanding of early pathogenetic mechanisms leading to joint damage is the most convincing rationale to continue research in this direction.

The authors declare no conflict of interest.

\section{References}

1. Buchbender C, Ostendorf B, Mattes-György K, et al. Synovitis and bone inflammation in early rheumatoid arthritis: high-resolution multi-pinhole SPECT versus MRI. Diagn Interv Radiol 2013; 19: 20-24.

2. Sudoł-Szopińska I, Kontny E, Zaniewicz-Kaniewska K, et al. Role of inflammatory factors and adipose tissue in pathogenesis of rheumatoid arthritis and osteoarthritis. Part I: Rheumatoid adipose tissue. J Ultrason 2013; 13: 192-201.

3. Smolen JS, Aletaha D, Steiner G. Does damage cause inflammation? Revisiting the link between joint damage and inflammation. Ann Rheum Dis 2009; 68: 159-162.

4. Miese FR, Ostendorf B, Wittsack H-J, et al. Metacarpophalangeal Joints in Rheumatoid Arthritis: Delayed Gadoliniumenhanced MR Imaging of Cartilage - A Feasibility Study. Radiology 2010; 257: 441-447.

5. Narváez JA, Narváez J, De Lama E, et al. MR imaging of early rheumatoid arthritis. Radiographics 2010; 30: 143-163. 
6. Sudoł-Szopińska I, Kontny E, Maśliński W, et al. The pathogenesis of rheumatoid arthritis in radiological studies. Part I: Formation of inflammatory infiltrates within the synovial membrane. J Ultrason 2012; 12: 202-213.

7. Hollander AP, Atkinst RM, Eastwoodt DM, et al. Human cartilage is degraded by rheumatoid arthritis synovial fluid but not by recombinant cytokines in vitro. Clin Exp Immunol 1991; 83: 52-57.

8. Gullick NJ, Evans HG, Church LD, et al. Linking power Doppler ultrasound to the presence of Th17 cells in the rheumatoid arthritis joint. PLoS One 2010; 5: e12516.

9. Bugatti S, Caporali R, Manzo A, et al. Involvement of subchondral bone marrow in rheumatoid arthritis: lymphoid neogenesis and in situ relationship to subchondral bone marrow osteoclast recruitment. Arthritis Rheum 2005; 52: 3448-3459.

10. Jimenez-Boy E, Redlich K, Türk B, et al. Interaction between synovial inflammatory tissue and bone marrow in rheumatoid arthritis. J Immunol 2005; 175: 2579-2588.

11. McQueen FM, Benton N, Perry D, et al. Bone edema scored on magnetic resonance imaging scans of the dominant carpus at presentation predicts radiographic joint damage of the hands and feet six years later in patients with rheumatoid arthritis. Arthritis Rheum 2003; 48: 1814-1827.

12. Tamai I, Kawakami A, Uetani M, et al. A prediction rule for disease outcome in patients with undifferentiated arthritis using magnetic resonance imaging of the wrists and finger joints and serologic autoantibodies. Arthritis Rheum 2009; 61: 772-778.

13. Sudoł-Szopińska I, Kontny R, Maśliński W, et al. Significance of bone marrow edema in pathogenesis of rheumatoid arthritis. Pol J Radiol 2013; 78: 57-63.

14. Manara M, Varenna M. A clinical overview of bone marrow edema. Reumatismo 2014; 66: 184-196.

15. Ostendorf B, Peters R, Dann P, et al. Magnetic resonance imaging and miniarthroscopy of metacarpophalangeal joints. Sensitive detection of morphologic changes in rheumatoid arthritis. Arthritis Rheum 2001; 44: 2492-2502.

16. Ostendorf B, Scherer A, Modder U, et al. Diagnostic value of magnetic resonance imaging of the forefeet in early rheumatoid arthritis when findings on imaging of the metacarpophalageal joints of the hands remain normal. Arthritis Rheum 2004; 50: 2094-2102.

17. McQueen FM, Ostendorf B. What is MRI bone oedema in rheumatoid arthritis and why does it matter? Arthritis Res Ther 2006; 8: 222-225.

18. Sudoł-Szopińska I, Zaniewicz-Kaniewska K, Warczyńska A, et al. Pathogenesis of rheumatoid arthritis in radiological studies. Part II: Imaging studies in rheumatoid arthritis. J Ultrason 2012; 12: 319-328.

19. Walsch DA. Angiogenesis and arthritis. Rheumatology 1999; 38: 103-112.

20. Clockaerts S, Bastiaansen-Jenniskens YM, Runhaar J, et al. The infrapatellar fat pad should be considered as an active osteoarthritic joint tissue: a narrative review. Osteoarthritis Cartilage 2010; 18: 876-882.

21. Ushiyama T, Chano T, Inoue K, et al. Cytokine production in the infrapatellar fat pad: another source of cytokines in knee synovial fluids. Ann Rheum Dis 2003; 62: 108-112.

22. Ostergaard M, Peterfy C, Conaghan P, et al. OMERACT rheumatoid arthritis magnetic resonance imaging studies: core set of MRI acquisitions, joint pathology definitions, and the OMERACT RA MRI scoring system. J Rheumatol 2003; 30: $1385-1386$

23. Oei EHG, Tiel J, Robinson WH, et al. Quantitative radiologic imaging techniques for articular cartilage composition: toward early diagnosis and development of disease-modifying therapeutics for osteoarthritis. Arthritis Care Res (Hoboken) 2014; 66: 1129-1141.

24. Jungius K-P, Schmid MR, Zanetti M, et al. Cartilaginous defects of the femorotibial joint: accuracy of coronal short inversion time inversion-recovery MR sequence. Radiology 2006; 240: 482-488.

25. Gagliardi JA, Chung EM, Chandnani VP, et al. Detection and staging of chondromalacia patellae: relative efficacies of conventional MR imaging, MR arthrography, and CT arthrography. Am J Roentgenol 1994; 163: 629-636.

26. Matzat SJ, van Tiel J, Gold GE, et al. Quantitative MRI techniques of cartilage composition. Quant Imaging Med Surg 2013; 3: 162-174.

27. Bittersohl B, Miese FR, Hosalkar HS, et al. T2* mapping of hip joint cartilage in various histological grades of degeneration. Osteoarthritis Cartilage 2012; 20: 653-660.

28. Bittersohl B, Hosalkar HS, Sondern $M$, et al. Spectrum of T2* values in knee joint cartilage at $3 \mathrm{~T}$ : a cross-sectional analysis in asymptomatic young adult volunteers. Skeletal Radiol 2014; 43: 443-452.

29. Tiel J, Kotek G, Reijman M, et al. Is T1r Mapping an Alternative to Delayed Gadolinium-enhanced MR Imaging of Cartilage in the Assessment of Sulphated Glycosaminoglycan Content in Human Osteoarthritic Knees? An in Vivo Validation Study. Radiology 2016; 279: 523-531.

30. Schleich C, Müller-Lutz A, Sewerin P, et al. Intra-individual assessment of inflammatory severity and cartilage composition of finger joints in rheumatoid arthritis. Skeletal Radiol 2015; 44: 513-518.

31. Buchbender C, Scherer A, Kröpil P, et al. Cartilage quality in rheumatoid arthritis: comparison of $\mathrm{T} 2{ }^{*}$ mapping, native $\mathrm{T} 1$ mapping, dGEMRIC, $\triangle R 1$ and value of pre-contrast imaging. Skeletal Radiol 2012; 41: 685-692.

32. Bittersohl B, Hosalkar HS, Miese FR et al. Zonal T2* and $\mathrm{T} 1_{\mathrm{Gd}}$ assessment of knee joint cartilage in various histological grades of cartilage degeneration: an observational in vitro study. BMJ Open 2015; 5: e006895.

33. Zilkens C, Miese FR, Crumbiegel $C$, et al. Magnetic resonance imaging and histology of ovine hip joint cartilage in two age populations: a sheep model with assumed healthy cartilage. Skeletal Radiol 2013; 42: 699-705.

34. Tiderius CJ, Sandin J, Svensson J, et al. Knee cartilage quality assessed with dGEMRIC in rheumatoid arthritis patients before and after treatment with a TNF inhibitor. Acta Radiol 2010; 51: 1034-1037.

35. Müller-Lutz A, Schleich C, Sewerin P, et al. Comparison of Quantitative and Semiquantitative Dynamic ContrastEnhanced MRI With Respect to Their Correlation to Delayed Gadolinium-Enhanced MRI of the Cartilage in Patients With Early Rheumatoid Arthritis. J Comput Assist Tomogr 2015; 39: 64-69. 
36. Miese F, Buchbender C, Scherer A, et al. Molecular Imaging of Cartilage Damage of Finger Joints in Early Rheumatoid Arthritis With Delayed Gadolinium-Enhanced Magnetic Resonance Imaging. Arthritis Rheum 2012; 64: 394-399.

37. Buchbender C, Sewerin P, Mattes-György K, et al. Utility of combined high-resolution bone SPECT and MRI for the identification of rheumatoid arthritis patients with high-risk for erosive progression. Eur J Radiol 2013; 82: 374-379. 